individual elements of the series from solution and the fused state, should be of great assistance to those wishing to prepare sources for radiochemical, physical, and biological use. A discussion of artificial radioelements and their electrochemical properties-a section necessarily short because of recent conditions -concludes this valuable book.

F. MORGAN

${ }^{1}$ J. chim. Phys., 32, 116 (1935).

- National Research Council of Canada. Montreal Laboratory Report MC.33.

\section{EMBRYOLOGY FOR MEDICAL STUDENTS}

Human Embryology

By Prof. Bradley M. Patten. Pp. xv + 776. (London : J. and A. Churchill, Ltd., 1946.) 45s.

ITTLE more than a year ago a new English text-book of human embryology had a somewhat mixed reception in these columns ${ }^{1}$. Criticisms were that it did not refer to the work of certain British men of science ; that a few features in some diagrams were incorrectly labelled; and that here and there the text could have been expanded to advantage. To-day another text-book with precisely the same title is addressed to medical students by Prof. Patten, a well-known and much esteemed American embryologist. It is slightly longer; slightly more lavishly, if not as well (although this is largely a matter of taste), illustrated; it covers almost the same ground; and doubtlessly it is open to some of the same criticism. It would be surprising if it were otherwise. In a subject like topographical embryology, where ideas on the genesis of organs represent dynamic concepts based on successive 'still' views of developmental processes, there is ample opportunity for differences in individual interpretation and emphasis ; and furthermore, so much scope that no one person could be expected to have a first-hand knowledge of every part of the whole field.

The purpose of Prof. Patten's book is to present to the medical student a conspectus of human morphogenesis. In this, like the English volume it follows, it succeeds admirably. If either were mastered the student would know far more about general embryology than a very high proportion of lecturers and demonstrators in British anatomical departments to-day-and by the same token very much more than his clinical teachers. No doubt undergraduates are already trying to do this, for the book of a year ago is already sold out, and this is almost certain to be the fate of Prof. Patten's very readable addition to the literature of embryology.

The very fact that this is so makes one ask whether it is right to expect the average medical student to attempt as much as is covered by Prof. Patten. Prof. Patten recognizes that the medical curriculum is overcrowded, and the consequent necessity to confine his book to a 'reasonable compass'. Anatomists who have specialized in embryology may agree with him that seven hundred pages of text is a reasonable compass; and others will as cordially argue that it is far too much. The difficulty here is that, to the man who has not made organogenesis a speciality, the case for devoting to the subject a large part of the anatomical curriculum often appears much overdone-and indeed at times pressed out of the bounds of all practical possibility. Protagonists of embryology offer the excuse that the subject helps to rationalize the topographical detail of anatomy; to understand the basis of the occasional abnormality met with in clinical practice ; to appreciate certain of the problems of obstetrics ; and above all, that it has a major intellectual or cultural value. Item by item few would bother to dispute any of this. Yet to state that a detailed understanding of the unfolding of a topographical pattern in the embryo is a basis for comprehending the detailed pattern in the adult is to come close to the statement of a tautology. To insist that a study of embryology helps the student to understand the occasional congenital abnormality met with in clinical practice is also to state the obvious; and suggests the logical conclusion that proportionately as little time should be devoted to the subject as is taken up in clinical practice by malformations. To state that the subject has a cultural value is merely to underline what should be a quality of the entire curriculum of the medical student. One may well ask oneself whether the whole pattern of these considerations is important in the educational problem which the average medical student constitutes.

For in any event the student has to take embryology very much as a matter of faith. In Great Britain he practically never examines a section of a human or pig embryo, still less a set of serial sections-among other good reasons, for the very important one that adequate study of such material is a very timeconsuming affair. Consequently the 'stills' and diagrams of his text-books represent the only direct experience the average student has of dynamic developmental processes. Such slender foundations scarcely sustain a scientific discipline. Even if they did, it is difficult to see what particular good it would be to the budding medical man. Books like Prof. Patten's are, in short, admirable guides for the student who has the opportunity and desire to study organogenesis in the laboratory itself. But they are much more than is wanted-to quote his own words-for the successful understanding of "the emergence of the various organ systems from undifferentiated primordial tissues to a point where he can recognize the beginnings of familiar adult structures"--which is about all one is likely to ask of the average medical student of to-day. What he wants is some short text that briefly explains what is known about the recognized principles of morphogenesis, and which is illustrated by really competent diagrams of the main stages in organogenesis. Since we are dealing with processes, and not fixed structures, it would be still better if one could call upon the talents of a first-class producer of 'animated films' to provide really good films of embryonic development. That would still be several steps from the original material of the subject; but it would be far closer than the student gets to-day.

For all this, there can be little doubt that Prof. Patten's main reading public will, in fact, be hurried medical students. One hopes, however, that it will receive more earnest employment as well. For topographical embryology does not need to be excused by over-emphasizing its utilitarian volue in medical education. It stands in its own right, as an essential part of the story of development-and without which morphology would be only half a science. From this point of view we may count ourselves as lucky that there are always some departments of anatomy that provide the subject with a secure home.

1 Nature, 156, 815 (1945). 